\title{
Research and Prediction of Wettability of Irregular Square Column Structure on Polymethyl Methacrylate (PMMA) Surface Prepared by Femtosecond Laser
}

\author{
Bangfu Wang $1,2, *\left(\mathbb{D}\right.$ and Juan Song ${ }^{1}$ \\ 1 College of Mechanical Engineering, Suzhou University of Science and Technology, Suzhou 215009, China; \\ songjuan@mail.usts.edu.cn \\ 2 College of Mechanical Engineering, Jiangsu University, Zhenjiang 212013, China \\ * Correspondence: bfwang@mail.usts.edu.cn; Tel.: +86-512-6832-0580
}

\section{check for}

updates

Citation: Wang, B.; Song, J. Research and Prediction of Wettability of Irregular Square Column Structure on Polymethyl Methacrylate (PMMA) Surface Prepared by Femtosecond Laser. Coatings 2021, 11, 529. https:// doi.org/10.3390/coatings11050529

Received: 21 March 2021

Accepted: 26 April 2021

Published: 29 April 2021

Publisher's Note: MDPI stays neutral with regard to jurisdictional claims in published maps and institutional affiliations.

Copyright: (c) 2021 by the authors. Licensee MDPI, Basel, Switzerland. This article is an open access article distributed under the terms and conditions of the Creative Commons Attribution (CC BY) license (https:// creativecommons.org/licenses/by/ $4.0 /)$.

\begin{abstract}
Based on the contact angle prediction model of a traditional square column structure, the prediction models for wettability of a parallelogram square column structure (PSCS) on polymethyl methacrylate (PMMA) surface prepared by femtosecond laser were established. An experiment was conducted to analyze the rationality of the established complete wetting model and incomplete wetting model. It was found that the incomplete wetting prediction model of the square column structure was more in line with the actual situation. For PSCS, the length of both the long and short sides of the boss and the width of the groove exerted an impact on the contact angle prediction results. Under the condition that the length of the long and short sides of the boss remained unchanged and the groove width increased, the contact angle increased under complete wetting and incomplete wetting. In contrast, under the condition that the long side length of the boss and the groove width remained unchanged and the short side length of the boss increased, the contact angle increased under complete wetting but decreased under incomplete wetting. The maximum contact angle reached $135.65^{\circ}$, indicating that PSCS on PMMA surface enhanced the surface hydrophobicity of the material.
\end{abstract}

Keywords: femtosecond laser; polymethyl methacrylate; hydrophobicity; parallelogram square column structure

\section{Introduction}

Polymethyl methacrylate (PMMA) is a typical polymer material widely used in various fields of human production and life [1]. It is often used in artificial bone, joint, and intraocular lens in biomedicine. The surface of PMMA has some defects, such as low surface energy, surface pollution, and weak boundary layer, which make it difficult for PMMA to wet and adhere [2,3]. Therefore, it is necessary to modify its surface to improve its surface properties. The wetting phenomenon exists in all fields of nature. For solid materials, wetting is one primary characteristic of the material surface [4], which has been used by humans in the fields of oil filtering, anti-pollution, lubrication, bonding, waterproofing, etc. [5-10]. When the liquid contacts a solid surface, a certain contact angle is formed. If the contact angle is not less than $90^{\circ}$, the liquid is considered to be non-wetting to the solid, and such solid surface is called lyophobic surface.

At present, researchers have proposed various methods for preparing surface wettability structures. Razavi et al. [11] prepared micro-modeling on the copper surface by chemical vapor deposition. Although various chemical methods have been widely used in the preparation of hydrophobic structures, micro- to nanostructures constructed by chemical methods have random properties, and the structural arrangement cannot be controlled artificially, which negatively affects hydrophobicity prediction of the materials. Cao et al. [12] prepared microstructures on the single crystal silicon surface by 
micro-milling. However, the properties of the processed microstructure are often subject to influence of machine tool chatter and tool wear, leading to processing quality problems such as chipping on the surface of the processed microstructure. Song et al. $[13,14]$ used a $0.1 \mathrm{~mm}$ diameter micro-milling cutter to machine the corresponding groove structure and square column array structure surface, measured the contact angle of water droplets on the machined surface, and compared it with the theoretical prediction value. They found that the groove and square column array structure can increase the contact angle of the water droplet on the surface, rendering a more stable contact angle change of the water droplet on the groove structure surface. However, the micro-milling processing efficiency is low, and the surface quality and accuracy have certain problems. Chen et al. [15] developed a rapid electrodeposition process and made a superhydrophobic cathode surface on a copper sheet placed in an electrolyte (nickel chloride, myristic acid, and ethanol). However, the methods are limited due to high cost of cumbersome process and the excessive time consumption. Although the chemical and mechanical processing methods mentioned above have been widely used to prepare hydrophobic surface microstructures, the wettability of some microstructures needs to be further improved, and the prediction law of contact angle needs to be further studied.

Recent years have witness rapid development of femtosecond laser processing technology. Scholars have applied laser processing to the preparation and modification of surface microstructures, which overcomes the size constraints and quality problems of traditional mechanically-processed surface microstructures [16,17]. Chichkov et al. [18] used femtosecond laser, picosecond laser, and nanosecond laser to drill holes on the steel surface. Researchers have found that the core mechanism of material removal under long-pulse laser irradiation is thermal deposition, and it is easy to form molten material on the structure edges after processing. Wang et al. [19] used laser to process the PMMA surface and prepare grating and square column microstructures and established a complete wetting model and an incomplete wetting model for experimental studies. They found that the microstructure had better hydrophobicity when the ratio of boss width to groove width was small and the spacing between the top edges was large. The processing mechanism of polymers is based on the breakage of chemical bond energy. After absorbing sufficient photon energy, the polymer chemical bonds will be broken, so that a microstructure will be formed. Femtosecond laser with ultra-high peak power and shorter reaction time can not only achieve a high removal rate of polymer materials but also ensure smoothness of the prepared micro- to nanostructures [20-23]. In addition, the focus of researchers has transited from single-layer structure to double-layer structure in order to achieve superhydrophobic effect of the material surface. Li [24] prepared a micro- to nanostructure on the material surface by changing a variety of processing parameters and optimized the processing parameters of superhydrophobic surface, finding that the surface hydrophobicity is higher when the micro- to nanostructure is more complex. Yong et al. [25] used femtosecond laser to construct micro well array structure on the surface of polydimethylsiloxane (PDMS) and achieved surface superhydrophobic performance by designing the surface micro well array size, spacing and other parameters [26].

The Wenzel model [27] and Cassie-Baxter model [28] are often used to predict the contact angle of micro- to nanostructures. At present, the research on droplet wetting behavior is mainly focused on regular nanostructures such as grid shape and square column array $[29,30]$. Saha et al. [31] studied the wetting characteristics of water droplets on graphite surfaces with different geometric parameters (column height, column width, and column spacing) and found that, under the optimal column spacing, compared with the wetting of water droplets on the surface of square column array, the wetting of water droplets on the surface of cylindrical array has higher fluidity. Bico et al. [32] discussed the wetting behavior of droplets on nanostructured surfaces and the possibility of different wetting state transitions. The results show that increasing column height and width and decreasing column spacing are beneficial to the formation of Cassie droplets. Drelich et al. [33] found that, when the actual surface structure of the object was in a protruding state, its parameters 
had a great impact on the stability of the hydrophobicity. The above results show that the traditional square column structures could be fabricated by laser, such as inverted trapezoid, square, and regular trapezoid microstructures. However, there is no in-depth study on the parameter variables of regular square structure. Hence, it is necessary to further improve the shape and size parameters of microstructure, introduce more variables, and analyze the influence of these parameters on the surface hydrophobicity of materials.

In this study, based on the contact angle wetting prediction model for Gibbs free energy square column structure, the complete wetting model and incomplete wetting model were designed and established for PSCS. In addition, the complete wetting model of the PSCS was numerically simulated to analyze the influence of its structural parameters on PMMA surface hydrophobicity. Finally, a femtosecond laser processing system was used to prepare PSCS with different structural parameters, and the effect of PMMA structural parameters on surface wettability was analyzed and verified based on numerical simulation results. The results suggest that the established prediction model is reasonable, and the structural parameters play a guiding role in improving surface structure hydrophobicity of the polymer PMMA.

\section{Establishment of Wetting Prediction Model for Parallelogram Square Column Structure}

\subsection{Incomplete Wetting Prediction Model}

Based on the Wenzel model and Cassie-Baxter model of the square column structure on the PMMA surface, an incomplete wetting model for the parallelogram square column structure (PSCS) was constructed. Figure 1 shows the constructed PSCS that conforms to laser characteristics.
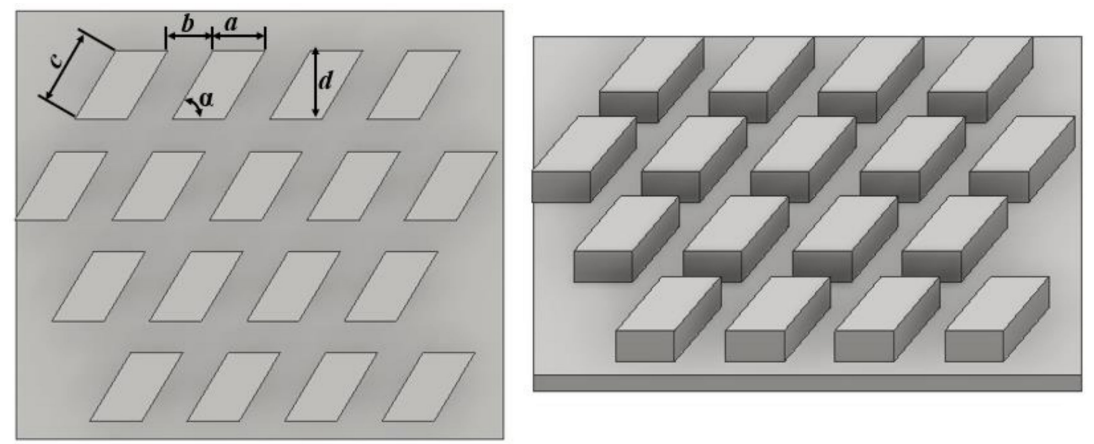

Figure 1. Schematic diagram of PSCS. $a$ represents the short side width of the designed PSCS, $b$ represents the width between the structure spacing (that is, the groove width), $c$ represents the long side width of the PSCS, $d$ represents the height of the short side width, and $\alpha$ is the small angle between the two adjacent sides of the PSCS. The PSCS has an intersection angle of $60^{\circ}$.

According to the actual wetting state of the PMMA material surface, a complete wetting model and an incomplete wetting model were established, as shown in Figure 2. When the droplet completely wets the material surface, the angle between the solid-liquid contact line and the gas-liquid contact line is defined as the contact angle $\theta^{\prime}$, as shown in Figure 2a. In the case of incomplete wetting model, the angle between the solid-liquid contact line and the gas-liquid contact line is defined as $\theta^{\prime \prime}$, as shown in Figure $2 \mathrm{~b}$. In addition, $r$ in the wetting model represents the radius of the contact surface between the droplet and the solid, while $R$ represents the radius of the spherical droplet. 

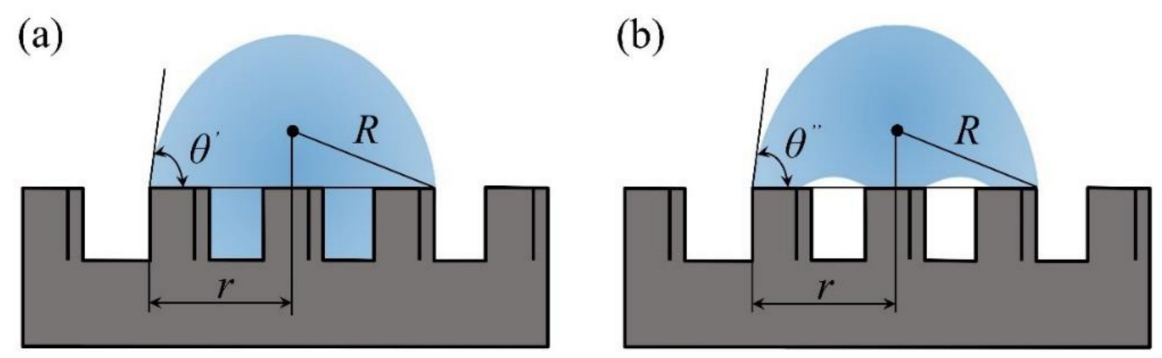

Figure 2. Wetting state of droplet on the surface structure of solid materials: (a) complete wetting model; and (b) incomplete wetting model.

According to the introduction of Gibbs free energy mentioned above, when the droplet is placed on the material surface in a stable state, its Gibbs free energy will display the smallest value. Therefore,

$$
d G=0
$$

When the droplet is placed on the material surface, an equilibrium state is shown, and the total surface area of the parallelogram square column boss below the droplet can be calculated as $S$ [19]:

$$
S=\frac{\pi r^{2}}{(2 r)^{2}} \times \frac{2 r}{d+b} \times \frac{2 r}{a+b} \times a \sin \alpha=\frac{a \pi r^{2} \operatorname{csin} \alpha}{(\mathrm{a}+\mathrm{b}) \times(\sin \alpha+b)}
$$

According to the parameters in Figures 1 and $2 b$, the solid-liquid and liquid-gas contact areas under the incomplete wetting prediction model can be expressed as $A_{s}$ and $A_{l v}$, respectively:

$$
\begin{gathered}
A_{s l}=S=\frac{a \pi r^{2} c \sin \alpha}{(a+b) \times(\sin \alpha+b)} \\
A_{l v}=\int_{0}^{\theta_{c}} 2 \pi r \mathrm{~d} \theta+\pi r^{2}-S=\frac{2 \pi r^{2}}{1+\cos \theta_{c}}+\pi r^{2}-\frac{a \pi r^{2} c \sin \alpha}{(a+b) \times(c \sin \alpha+b)}
\end{gathered}
$$

Since the solid-gas contact area cannot be directly expressed, the surface area of the PSCS is introduced to indicate the interface area:

$$
A_{s v}=A_{s}-A_{s l}
$$

By combining the above formulas,

$$
\cos \theta_{c}=\frac{\gamma_{s v}-\gamma_{s l}}{\gamma_{l v}}=\frac{a \sin \alpha(\cos \theta+1)}{(a+b) \times(\operatorname{csin} \alpha+b)}-1
$$

where $\gamma_{\mathrm{sv}}, \gamma_{\mathrm{sl}}$, and $\gamma_{\mathrm{lv}}$ are the interfacial free energy of solid-gas, solid-liquid and liquid-gas interfaces, respectively.

Before selecting the appropriate grating structure parameters, theoretical derivation is needed to predict the relationship between the contact angle and the geometric parameters, and Matlab is used to analyze the geometric parameters and contact angle of the grating structure under the incomplete wetting model. According to Formula (6), when the PSCS has constant depth, the variation trends of the width $a$, the spacing $b$ between the bosses and the contact angle of the PSCS under the incomplete wetting prediction model are shown in Figure 3. 


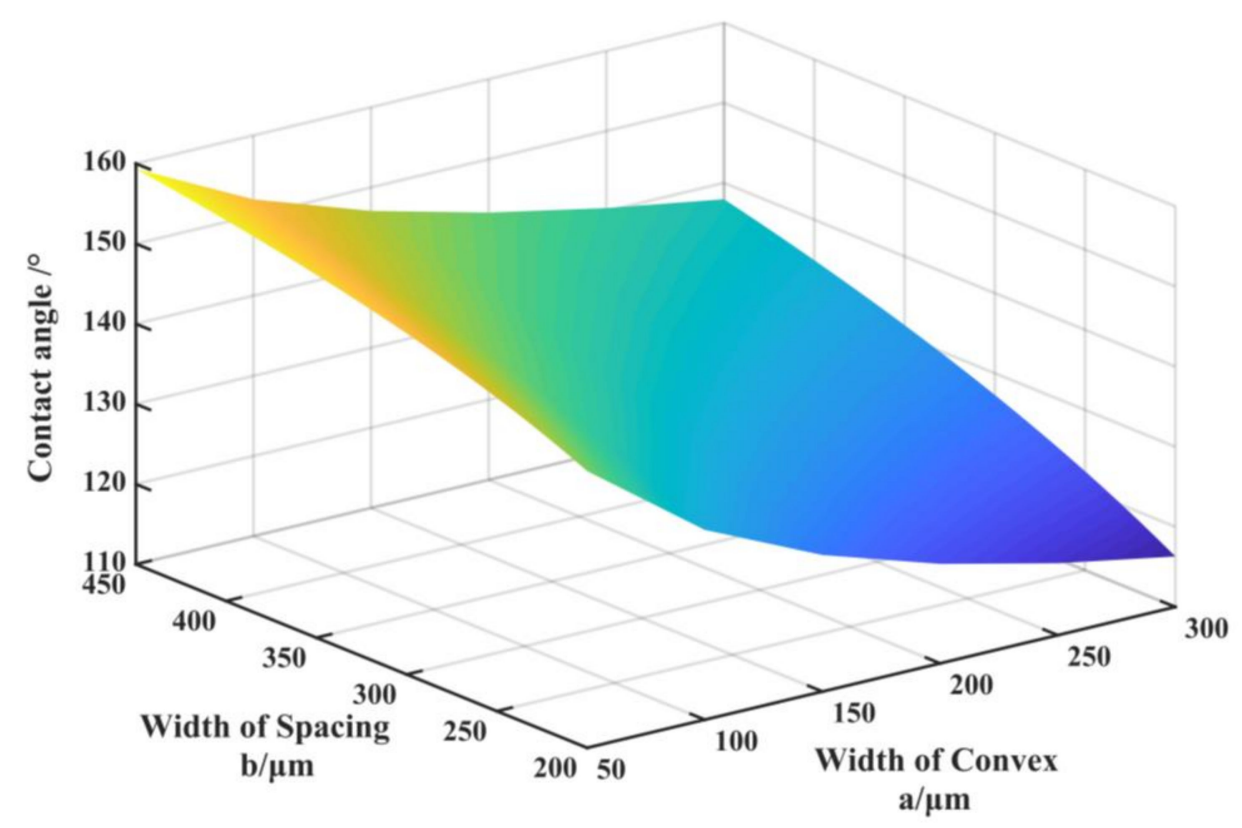

Figure 3. Three-dimensional relationship curve of contact angle and structural parameters under the incomplete wetting prediction model.

As shown in Figure 3, under smaller boss width of the PSCS, the greater is the boss spacing, the bigger is the predicted contact angle value. According to the single factor variable, it can be seen that, under smaller boss width of the PSCS, the predicted contact angle value is bigger. In addition, under greater boss spacing in the square column structure, the predicted contact angle value is bigger.

\subsection{Complete Wetting Prediction Model}

In the complete wetting prediction model, the contact area between the gas phase and the liquid phase differs greatly from that under the incomplete wetting prediction model. $h$ is the groove depth of the PSCS. The solid-liquid and liquid-gas phase contact area under the complete wetting prediction model can be expressed as $A_{s l}$ and $A_{l v}$, respectively:

$$
\begin{gathered}
A_{s l}=\pi r^{2}+\frac{\pi r^{2}(2 a h+2 c h)}{(a+b) \times(\sin \alpha+b)} \\
A_{l v}=\int_{0}^{\theta_{w}} 2 \pi r \mathrm{~d} \theta=\frac{2 \pi r^{2}}{1+\cos \theta_{w}}
\end{gathered}
$$

The solid-gas contact area is $A_{s v}=0$, thus

$$
\cos \theta_{w}=\left[1+\frac{2 a h+2 c h}{(a+b) \times(\operatorname{csin} \alpha+b)}\right] \cos \theta
$$

According to the above formula, the three-dimensional relationship curve of the boss width $a$, the boss spacing $b$, and the contact angle of the PSCS under the complete wetting prediction model can be numerically simulated, as shown in Figure 4. 


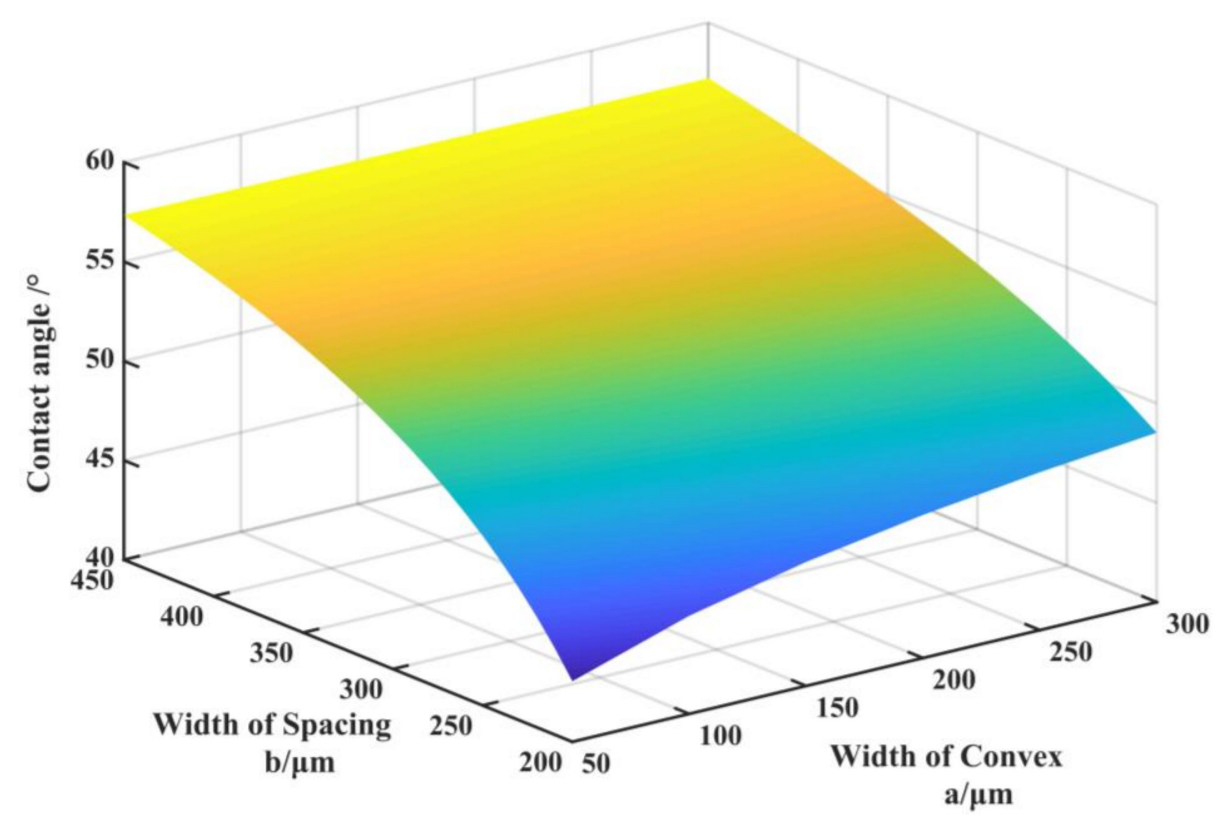

Figure 4. Three-dimensional curve of contact angle and square column structure parameters under the complete wetting model.

As shown in Figure 4, when the boss width of the PSCS is big or the top edge length of the spacing between the bosses of the PSCS enlarges, the contact angle will gradually increase. Moreover, boss spacing has a bigger influence on the contact angle than boss width.

\section{Experiment and Verification}

\subsection{Experimental Processing System}

The Origami-10XP femtosecond laser (Onefive GmbH, Zurich, Switzerland) produced by Swiss Onefive was used to prepare multi-level microstructures. The schematic diagram of the multistage microstructure machining system for PMMA surface prepared by femtosecond laser [19] is shown in Figure 5.

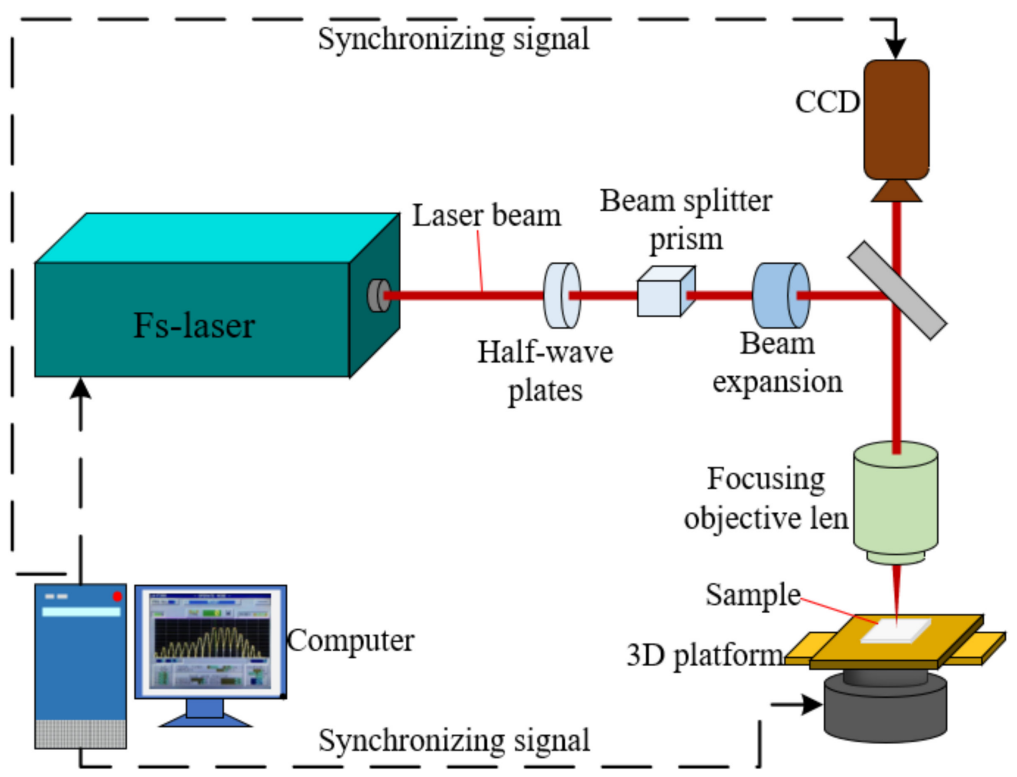

Figure 5. The principle diagram of the microstructure processing system prepared by femtosecond laser on PMMA surface. 
The laser output beam energy used in the experiment followed Gaussian distribution, and its main technical parameters are shown in Table 1. The key optical element of this experimental system is the focusing microscope objective lens, which determines the focusing spot size and the minimum machining precision. The microscope objective lens of the Japanese Mitutoyo was adopted in this experiment. The processing system has a laser output power of $4 \mathrm{~W}$, pulse width of $400 \mathrm{fs}$, and wavelength of $1030 \mathrm{~nm}$. The incident laser light is linearly polarized.

Table 1. Technical parameters of the femtosecond laser.

\begin{tabular}{ccccc}
\hline No. & Parameter Name & Symbol & Value & Unit \\
\hline 1 & Pulse width & $\tau$ & 400 & $\mathrm{fs}$ \\
2 & Wavelength & $\lambda$ & 1030 & $\mathrm{~nm}$ \\
3 & Maximum repetition frequency & $f$ & 1 & $\mathrm{MHz}$ \\
4 & Minimum repetition frequency & $f$ & 100 & $\mathrm{kHz}$ \\
5 & Maximum average power & $P$ & 4 & $\mathrm{~W}$ \\
6 & Maximum pulse energy & $E_{P}$ & 40 & $\mu \mathrm{J}$ \\
\hline
\end{tabular}

The precision processing system used in this experiment consists of a high-precision three-dimensional processing platform and a control system based on the motion controller (SPiiPlusEC, ACS, Migdal Ha-Emek, Israel). The entire laser processing system was monitored in real time by a CCD camera (MER-500-7-UC-L, China Daheng Group Co. Ltd., Beijing, China). The femtosecond laser beam was adjusted through the optical path, and then focused on the surface of the PMMA sample by the objective lens to ablate the PMMA sample. As the microstructure of the femtosecond laser-processed PMMA surface was on the micrometer scale, the accuracy of the 3D mobile platform directly determines the processing quality of the surface microstructure. The sample movement is controlled by the 3D mobile platform, and the laser spot position on the sample varied. The threedimensional mobile platform was controlled by a computer to achieve precise control of the processing structure. The $x$-axis and $y$-axis have an effective stroke of $50 \mathrm{~mm}$ and repeatability of $\pm 0.5 \mu \mathrm{m}$, while the vertical movement speed of the platform is $50 \mu \mathrm{m} / \mathrm{s}$.

\subsection{Processing Materials and Process Parameters}

According to the results of the prediction model in the previous section, appropriate structure parameters were selected, as shown in Table 2.

Table 2. Dimensional parameters of samples processed by femtosecond laser.

\begin{tabular}{cccc}
\hline Sample Number & Boss Width $\boldsymbol{a}(\boldsymbol{\mu m})$ & Boss Spacing $\boldsymbol{b}(\boldsymbol{\mu m})$ & Boss Width $\boldsymbol{c}(\boldsymbol{\mu m})$ \\
\hline 1 & 50 & 250 & 300 \\
2 & 250 & 250 & 420 \\
3 & 100 & 200 & 300 \\
4 & 150 & 200 & 300 \\
5 & 200 & 300 & 420 \\
6 & 200 & 400 & 420 \\
\hline
\end{tabular}

The material used in the verification experiment herein is polymethyl methacrylate (PMMA) with a dimension of $600 \mathrm{~mm} \times 400 \mathrm{~mm} \times 3 \mathrm{~mm}$. The surface of PMMA was polished and then cleaned with anhydrous ethanol and deionized water to remove the surface impurities. In the experiment, the laser beam had an output power of $3 \mathrm{~W}$ and processing speed of $50 \mathrm{~mm} / \mathrm{s}$. The laser beam adopted single-stroke and reciprocating processing in microstructure preparation.

\subsection{Observation of the Processing Samples}

After preparation of the PMMA surface multi-level microstructure using the femtosecond laser processing system, the prepared experimental sample was placed in an 
ultrasonic cleaner (JP-010T, Skymen, Shenzhen, China) with alcohol for 5 min to remove the surface impurities. Then, deionized water was used to remove residual alcohol solution on the PMMA surface. After that, metallographic tape was added to cover and paste on the PMMA surface to ensure accuracy of the subsequent measurement data.

In this paper, the surface hydrophobicity of the PMMA multi-level microstructure sample was measured with an optical contact angle measuring instrument (OCA, DataPhysics, Filderstadt, Germany). Attention was paid to the sample surface cleanness during each measurement in the cleanroom, and then computer software was used to control the $2 \mu \mathrm{L}$ deionized water in the injection needle, making it drop smoothly onto the PMMA multi-level microstructure surface for measurement of the contact angle. It is also necessary to ensure accuracy of the injection droplets. The contact angle measuring instrument had a measurement accuracy of $\pm 0.1^{\circ}$. To ensure accuracy of the contact angle measurement data and reduce the randomness of the measurement data, five data distribution points were randomly measured for a large area of the PMMA surface microstructure. The intrinsic contact angle of PMMA was measured by DataPhysics OCA optical contact angle measuring instrument (DataPhysics Instruments $\mathrm{GmbH}$, Filderstadt, Germany), which was $64^{\circ}$.

In addition, the super-high-magnification lens zoom 3D microscope (VHX-5000, Keyence, Osaka, Japan) was used to observe the surface morphology of the PMMA sample after processing. The partial morphology of the microstructure of the parallelogram square column on the PMMA surface at a magnification of 50 times is shown in Figure 6.

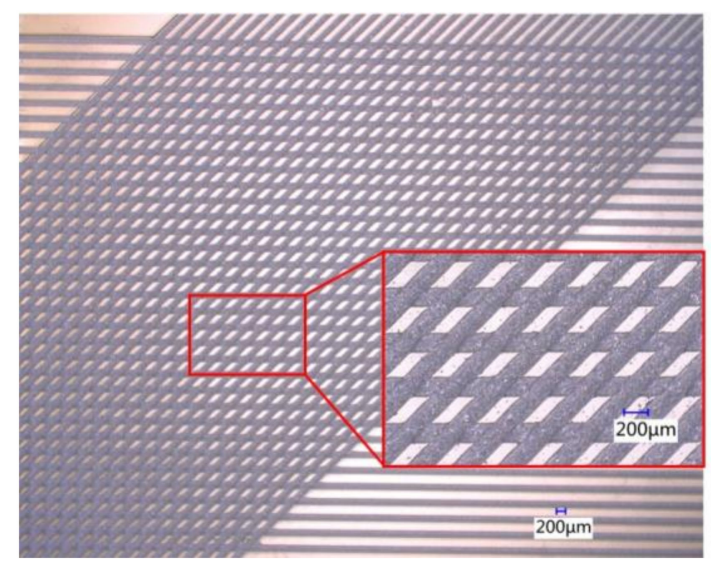

Figure 6. The microtopography of PSCS on the PMMA surface $(\times 50)$ : Sample 4.

\section{Results and Discussion}

The morphologies of PSCS on the PMMA surface after the processing were analyzed, as shown in Figure 7.

Figure 7 shows that, after preparation of PSCS on the PMMA surface, certain protrusions appear on the bosses of the structure surface. This is because the femtosecond laser beam has a Gaussian distribution and the laser pulse repetition frequency is much greater than the critical frequency, which led to accumulation of laser heat and thermal degradation of PMMA. Some of the degradation products escaped in the form of gas and some were condensed on the top of the boss. The contact angle measurement principle of the PMMA surface after microstructure preparation by laser processing is shown in Figure 8. 


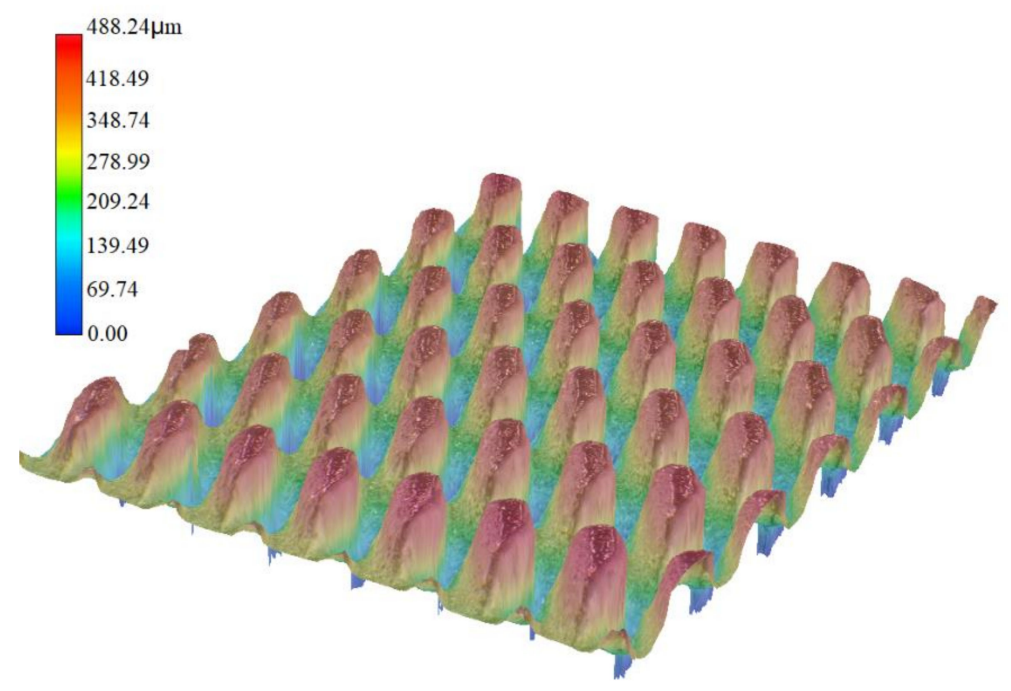

Figure 7. The three-dimensional topography of the PSCS on the PMMA surface.

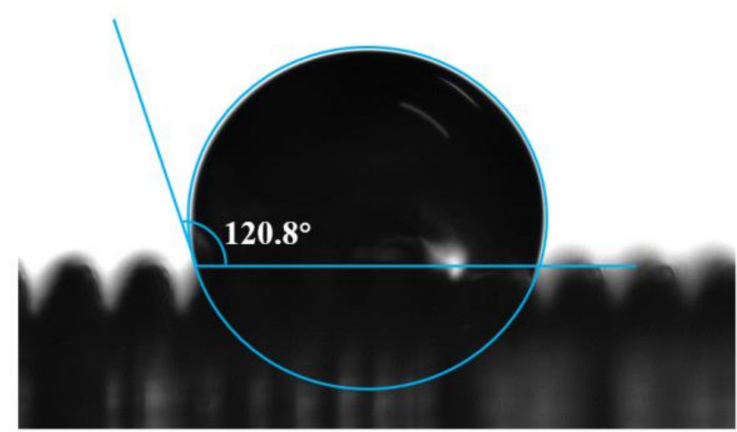

Figure 8. Experimental value of contact angle of PMMA surface after microstructure preparation by laser: Sample 4.

Using the contact angle measuring instrument, the experimentally measured value of contact angle of the sample was compared with the predicted value (Table 3).

Table 3. The predicted and experimental values of the contact angle of the PSCS on the PMMA surface.

\begin{tabular}{cccc}
\hline Sample Number & Experiment $\left(^{\circ}\right)$ & Complete Wetting Prediction Model $\left.^{\circ}{ }^{\circ}\right)$ & Incomplete Wetting Prediction Model $\left({ }^{\circ}\right)$ \\
\hline 1 & $135.44 \pm 2.80$ & 50.28 & 151.4 \\
2 & $114.35 \pm 0.93$ & 50.96 & 125.0 \\
3 & $128.27 \pm 1.30$ & 46.16 & 136.8 \\
4 & $120.08 \pm 2.51$ & 46.88 & 130.7 \\
5 & $125.85 \pm 0.54$ & 52.97 & 133.2 \\
6 & $131.72 \pm 1.01$ & 56.15 & 140.5 \\
\hline
\end{tabular}

Figure 9 shows the variation curve of the contact angle of the PSCS with boss width.

Figure 9 shows that, under the incomplete wetting model, the variation of predicted contact angle of the PSCS sample was consistent with that of the measured value. The measured contact angles of PSCS are all greater than $90^{\circ}$, which indicates that PMMA wettability can be modified by laser processing technology to improve hydrophobicity of the material surface. The measured contact angle of PSCS within the error range is quite close to the predicted value of contact angle under the incomplete wetting model. This was mainly due to an air layer between the water droplet and the microstructure under the incomplete wetting model, which made the water droplet hardly filled in the microstructure, resulting in small contact area between the droplet and the microstructure. This further suggests that 
the incomplete wetting model is more reasonable and accurate. Through comparing the intrinsic contact angle $64^{\circ}$ of PMMA surface, it can be observed that preparation of the microstructure on the PMMA surface can enable shift from hydrophilicity to hydrophobicity.

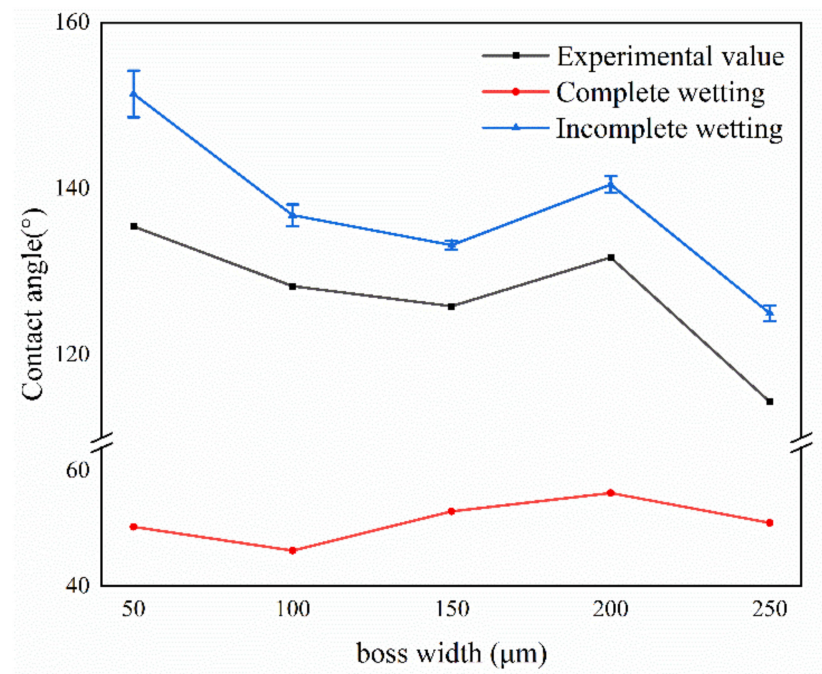

Figure 9. The variation curve of the contact angle of the PSCS with boss width.

Through comparing the curves of predicted and measured contact angle under incomplete wetting model, it can be observed that contact angle was bigger under smaller boss width of PSCS. Under boss width of $50 \mu \mathrm{m}$, the contact angle reaches the maximum of $135.65^{\circ}$. From the contact angle prediction model and experimental results, it can be found that, when boss width $a$ remains unchanged, boss spacing $b$ increases, and the contact angle increases gradually. In addition, when boss spacing $b$ remains unchanged, boss width $a$ decreases, and the contact angle increases gradually. It was found that the contact angle is close to the maximum value measured in the experiment and meets the theoretical prediction model. Hence, hydrophobicity of polymethyl methacrylate surface can be increased by reducing the boss width. In addition, the experimentally measured contact angle is always smaller than the contact angle predicted by the incomplete wetting model. As shown in Figure 9, the average difference between the measured value and predicted value by the incomplete wetting model is $10.315^{\circ}$. In contrast, the difference between the measured value and the predicted value by incomplete wetting model can reach $15.75^{\circ}$ under small boss width. The predicted contact angle of the incomplete wetting model in this paper is more than $10^{\circ}$ larger than that of the previous model. It is theoretically proved that the wettability of the structure designed in this paper can be improved. However, the predicted contact angle of the complete wetting model of PSCS is the same as that of the trapezoidal groove structure (TGS). Besides, the experimental values of both are consistent with the predicted values of the incomplete wetting model. The experimental average contact angle of PSCS is larger than that of TGS.

To further analyze the error between the measured and predicted values, the morphology of PSCS on the PMMA surface was analyzed using three-dimensional microscope with an ultra-high magnification lens. The three-dimensional morphology and cross section of the measured sample are shown in Figure 10.

Figure 10a shows that there were some protrusions on the boss surface of the PSCS on the PMMA surface, and the top length spacing of the groove is slightly longer than the theoretically designed spacing. Based on the actual measured contact angle data, it can be found that the larger is the distance between the bosses, the larger is the contact angle and the better is the hydrophobicity of the material. In fact, the protruding part of the boss surface of PSCS is a result of the interaction between the laser and PMMA. When the laser focus irradiates the bottom of the square column groove, the energy absorbed by the upper material of the PMMA is smaller than the absorption threshold of the PMMA 
material, which causes the lower material to decompose and produce internal stress. Then, the focus position will gradually move upward, with deformation caused by the internal stress gradually moved upward, and finally protrusions will appear on the boss surface of the PSCS [34-37]. The groove steepness of PSCS in the horizontal direction of the long side is larger than that in the vertical direction of the short side, as shown in Figure 10b. This was due to that the selected processing path involves repeated processing on both sides of the boss. Hence, the groove depth at the intersection of the parallel direction of the long side and the parallel direction of the short side is larger than that of the single side of the boss, but the distance between the boss is basically the same, indicating that femtosecond laser still has the advantage of processing in the smaller heat affected zone.
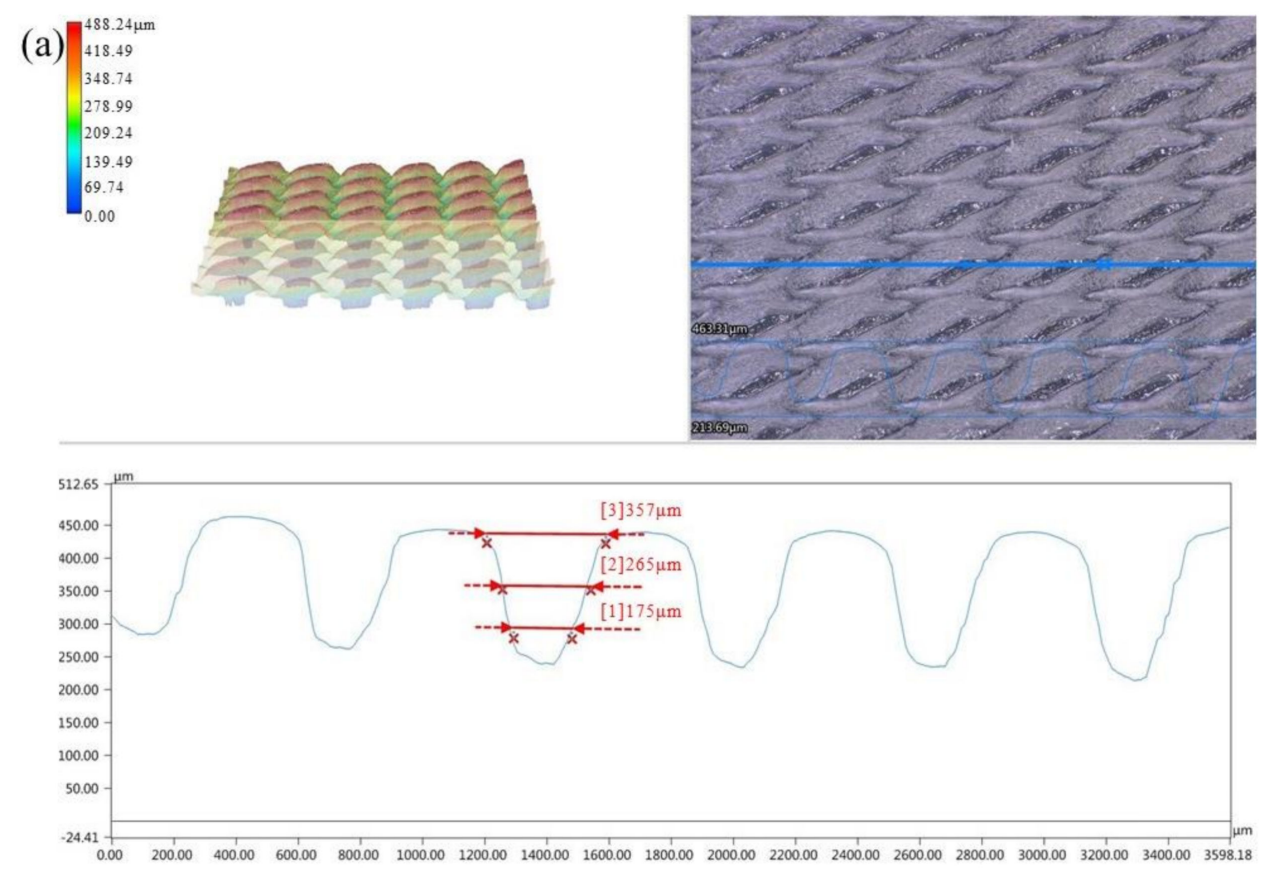

(b)

$488.24 \mu \mathrm{m}$
418.49
348.74
278.99
209.24
139.49
69.74
0.00
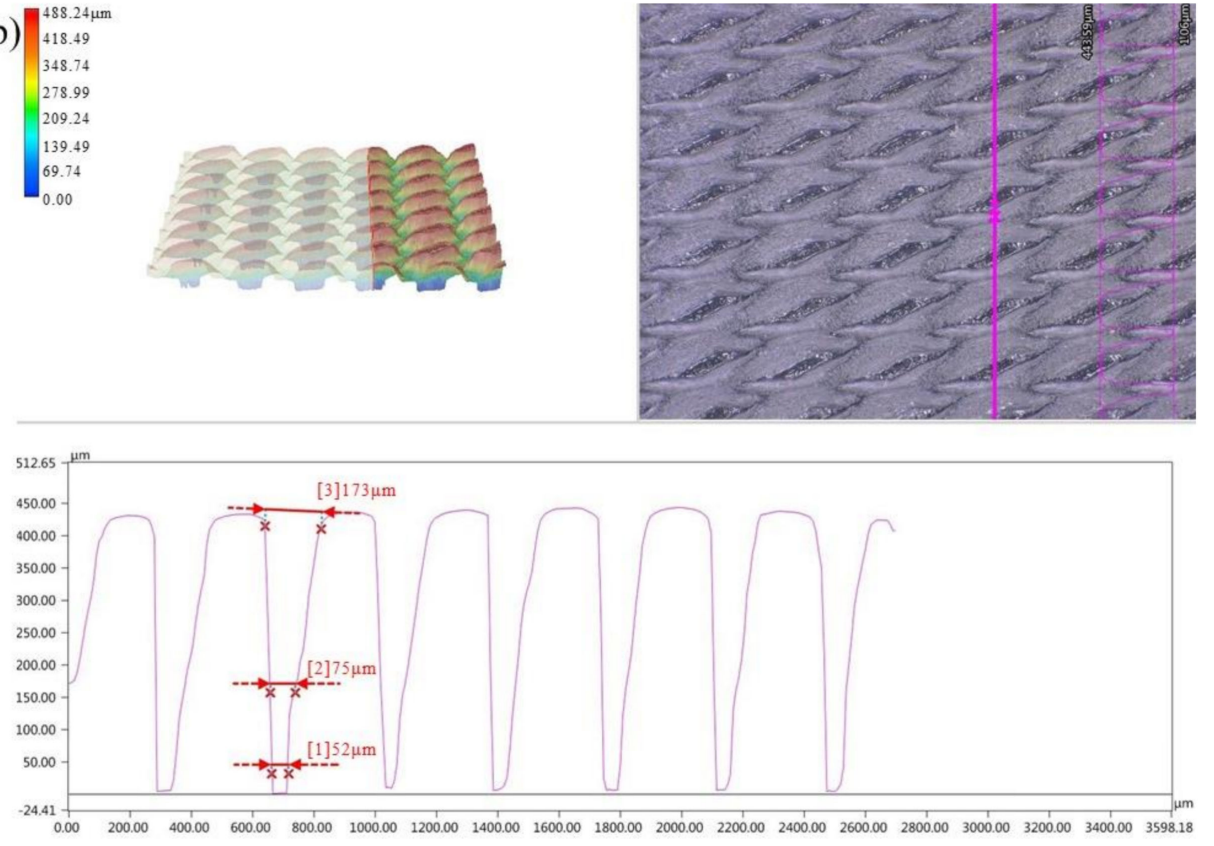

Figure 10. The three-dimensional morphology and cross section of the PSCS $(\times 100)$ : (a) along the height direction of the long side; and (b) along the height direction of the short side. 
To compare processing quality and wetting performance of the PSCS prepared by laser under different structural parameters, the three-dimensional profile cross-sectional curve distribution and the corresponding contact angle of the PSCS under different boss widths were obtained, as shown in Figure 11.
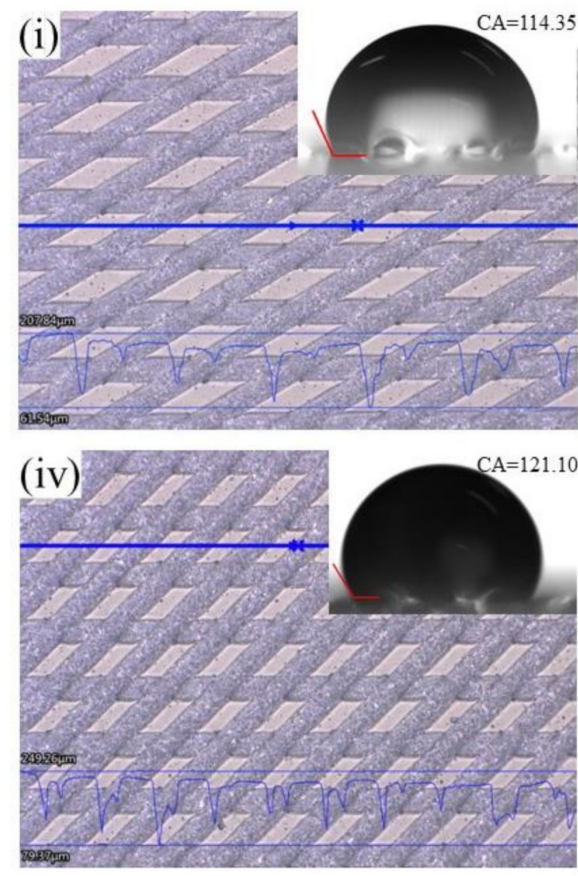
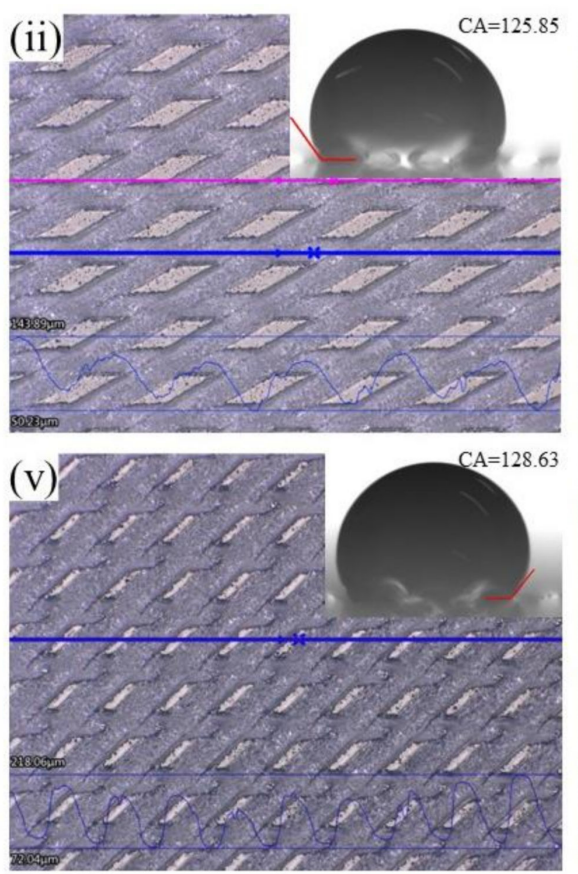
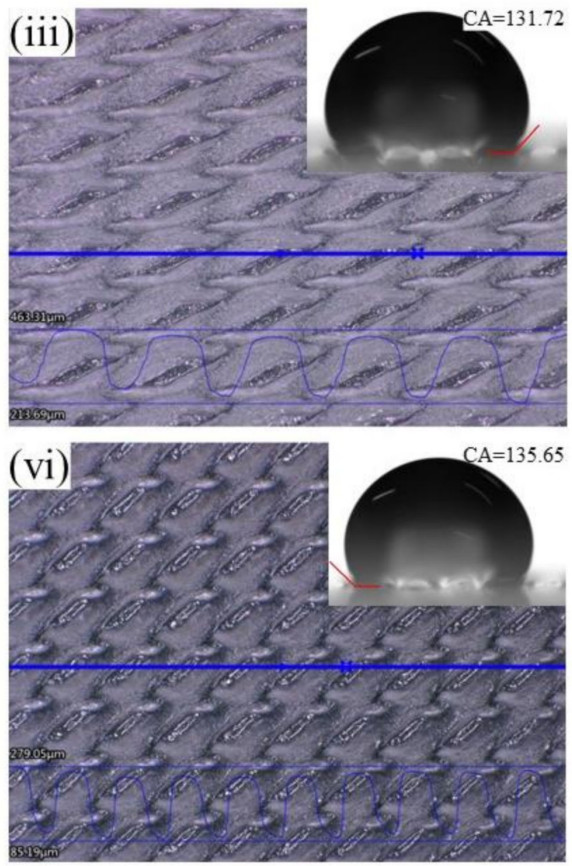

Figure 11. The three-dimensional profile cross-sectional curve distribution and the corresponding contact angle of the PSCS under different boss widths: (i) Sample 1; (ii) Sample 2; (iii) Sample 3; (iv) Sample 4; (v) Sample 5; and (vi) Sample 6.

Figure 11i-iv shows that, when the boss spacing and the long side length of the parallelogram square column remain constant, the contact angle decreased with the decrease of boss width. Since the boss width was too small, the laser beam followed Gaussian distribution, which resulted in certain inverted trapezoidal structure of the processed square column microstructure groove, causing certain deviation from the theoretically designed rectangular structure. As shown in Figure 11v,vi in the case of constant boss width, the contact angle of the parallelogram square column microstructure increased with the increase of boss spacing. This is because the contact area between the droplet and the microstructure surface decreases with decreased microstructure surface roughness as the boss spacing increases. According to the results of the above six sets of comparative experiments, it was found that, in the case of big boss width and small boss spacing, there was a ridge at the bottom of the microstructure groove, which affected the quality of prepared microstructure. Compared with the contact angle under several other structural parameters, the actual contact angle is small, which is due to that the ridge defect in the middle of the groove between the bosses causes greater contact area between the droplet and the microstructure, leading to somewhat reduced contact angle.

\section{Conclusions}

In this paper, the microstructure of the parallelogram square column structure was designed on PMMA surface, and the prediction model of complete wetting and incomplete wetting of the parallelogram square column structure was established. The parallelogram square column structure was fabricated on PMMA surface by femtosecond laser processing technology, and the accuracy of the model was verified by experiments.

Through numerical analysis of the contact angle prediction model and related experimental research, it was found that, compared with the complete and incomplete wetting prediction models, the incomplete wetting prediction model constructed in this work was 
more in line with the actual situation. For the parallelogram square column structure, the length of the long and short sides of the boss and the width of groove exerted an impact on the prediction results of contact angle. When the length of the long and short sides of the boss remained unchanged, the contact angle increased with the increase of groove width under complete and incomplete wetting models. In contrast, when the long side length of the boss and the groove width both remained unchanged, while the short side length of the boss increased, the contact angle increased under complete wetting model but decreased under incomplete wetting model. The maximum contact angle can reach $135.65^{\circ}$. There is error between the predicted value of incomplete wetting model and the experimental value. Due to the existence of a certain thermal ablation zone, the size parameters of the parallelogram square column structure after cooling and condensation have errors, which affect the hydrophobicity of PMMA surface.

In general, the wettability of the material surface can be affected by changing the structure and size parameters of parallelogram square column structure. In the future, according to the angle change of parallelogram square column structure, a double-layer structure or specific pattern can be constructed on the parallelogram square column structure to study its wettability.

Author Contributions: Writing — original draft preparation, B.W.; software and data curation, J.S.; writing-review and editing, B.W.; and methodology and formal analysis, B.W. All authors have read and agreed to the published version of the manuscript.

Funding: This research was funded by the National Key Research and Development Program, grant number 2018YFB1107700.

Institutional Review Board Statement: Not applicable.

Informed Consent Statement: Not applicable.

Data Availability Statement: All data, models, or code generated or used during the study are available from the corresponding author by request.

Conflicts of Interest: The authors declare no conflict of interest.

\section{References}

1. Hu, S.; Wang, C.; Cao, J.; Zhou, H.; Zhang, L.; Zhang, L.; Zhao, S. Effect of Zwitterionic Surfactants on Wetting of Polymethylmethacrylate Surface. In Proceedings of the 15th Conference on Colloid and Interface Chemistry of Chinese Chemical Society, Wuhan, Hubei, China, June-July 2015; pp. 135-136.

2. Lin, N.; Kim, M.; Lewis, G.; Cohen, Y. Polymer surface nano-structuring of reverse osmosis membranes for fouling resistance and improved flux performance. J. Mater. Chem. 2010, 20, 4642-4652. [CrossRef]

3. Sun, Y. Study on Surface Modification of Biomedical Polystyrene Film by Plasma. Ph.D. Thesis, Shandong University, Jinan, China, 2011.

4. Sun, L.; Li, M. Preparation and Properties of Chitosan-SiO ${ }_{2}$ Composite Films with Underwater Superoleophobicity. J. Acta Polym. Sin. 2014, 6, 822-830.

5. Su, B.; Tian, Y.; Jiang, L. Bioinspired interfaces with superwettability: From materials to chemistry. J. Am. Chem. Soc. 2016, 138, 1727-1748. [CrossRef] [PubMed]

6. Boreyko, J.B.; Collier, C.P. Delayed frost growth on jumping-drop superhydrophobic surfaces. ACS Nano 2013, 7, 1618-1627. [CrossRef]

7. Wang, S.; Liu, K.; Yao, X.; Jiang, L. Bioinspired surfaces with superwettability: New insight on theory, design, and applications. Chem. Rev. 2015, 115, 8230-8293. [CrossRef]

8. Fang, Y.; Zhou, M.; Li, M.; Miao, X.; Pei, Y.; Yan, Y.; Wu, L. On the Preparation Methods of Superhydrophobic Transparent Coatings. J. Hangzhou Norm. Univ. 2020, 19, 239-243. (In Chinese)

9. Wang, J.; Shi, X.; Feng, L.; Qiang, X.; Liu, Y. Construction of Long-acting Superhydrophobic Copper Surface and Its Wear Resistance and Self-cleaning Performance. Mater. Rev. 2018, 32, 4314-4318.

10. Shen, Q.; Wang, T.; Liang, J.; Wen, L. Hydrodynamic performance of a biomimetic robotic swimmer actuated by ionic polymermetal composite. Smart Mater. Struct. 2013, 22, 1-13. [CrossRef]

11. Razavi, S.M.; Oh, J.; Sett, S.; Feng, L.; Yan, X.; Hoque, M.J.; Liu, A.; Haasch, R.T.; Masoomi, M.; Bagheri, R.; et al. Superhydrophobic Surfaces Made from Naturally Derived Hydrophobic Materials. ACS Sustain. Chem. Eng. 2017, 5, 11362-11370. [CrossRef]

12. Cao, Z.; Ding, W.; Ma, Z.; Wang, B.; Wang, Z. Research on the Hydrophobicity of Square Column Structures on Monocrystalline Silicon Fabricated Using Micro-Machining. Micromachines 2019, 10, 763. [CrossRef] 
13. Song, H.; Liu, Z.; Shi, Z.; Cai, Y. Model of contact angle of hydrophobic surface based on minimum Gibbs free energy. J. Shandong Univ. 2015, 45, 56-61. (In Chinese)

14. Song, H. Investigations on Design and Micro-Milling of Micro-textured Hydrophobic Surface. Ph.D. Thesis, Shandong University, Jinan, China, 2015.

15. Chen, Z.; Hao, L.; Chen, A.; Song, Q.; Chen, C. A rapid one-step process for fabrication of superhydrophobic surface by electrodeposition method. Electrochim. Acta. 2012, 59, 168-171. [CrossRef]

16. Ta, D.V.; Dunn, A.; Wasley, T.J.; Kay, R.W.; Stringer, J.; Smith, P.J.; Connaughton, C.; Shephard, J.D. Nanosecond laser textured superhydrophobic metallic surfaces and their chemical sensing applications. Appl. Surf. Sci. 2015, 357, 248-254. [CrossRef]

17. Chen, M.; Ding, W.; Cheng, J.; Yang, H.; Liu, Q. Recent Advances in Laser-Induced Surface Damage of KH2PO4 Crystal. Appl. Sci. 2020, 10, 6642. [CrossRef]

18. Chichkov, B.N.; Momma, C.; Nolte, S.; von Alvensleben, F.; Tünnermann, A. Femtosecond, picosecond and nanosecond laser ablation of solids. Appl. Phys. A 1996, 63, 109-115. [CrossRef]

19. Wang, B.; Zhang, Y.; Song, J.; Wang, Z. Investigation and Prediction on Regulation of Hydrophobicity of Polymethyl Methacrylate (PMMA) Surface by Femtosecond Laser Irradiation. Coatings 2020, 10, 386. [CrossRef]

20. Farshchian, B.; Gatabi, J.R.; Bernick, S.M.; Lee, G.-H.; Droopad, R.; Kim, N. Scaling and mechanism of droplet array formation on a laser-ablated superhydrophobic grid. Colloids Surf. A 2018, 547, 49-55. [CrossRef]

21. Waugh, D.; Lawrence, J. On the use of $\mathrm{CO}_{2}$ laser induced surface patterns to modify the wettability of Poly (methyl methacrylate) (PMMA). Opt. Lasers Eng. 2010, 48, 707-715. [CrossRef]

22. Zhang, C.; Cao, M.; Ma, H.; Yu, C.; Li, K.; Yu, C.; Jiang, L. Morphology-Control Strategy of the Superhydrophobic Poly (Methyl Methacrylate) Surface for Efficient Bubble Adhesion and Wastewater Remediation. Adv. Funct. Mater. 2017, 27, 1702020. [CrossRef]

23. Li, J.; Wang, W.; Mei, X.; Pan, A.; Sun, X.; Liu, B.; Cui, J. Artificial Compound Eyes Prepared by a Combination of Air-Assisted Deformation, Modified Laser Swelling, and Controlled Crystal Growth. ACS Nano 2019, 13, 114-124. [CrossRef]

24. Li, F. Superhydrophobic Surfaces Fabricated by Femtosecond Laser Pulses and Study on Their Wettability. Ph.D. Thesis, Jilin University, Jilin, China, 2019.

25. Yong, J.; Chen, F.; Yang, Q.; Zhang, D.; Bian, H.; Du, G.; Si, J.; Meng, X.; Hou, X. Controllable adhesive superhydrophobic surfaces based on PDMS microwell arrays. Langmuir 2013, 29, 3274-3279. [CrossRef]

26. Bai, X.; Chen, F. Recent Advances in Femtosecond Laser-Induced Superhydrophobic Surfaces. Acta Opt. Sin. $2021,41,218-231$.

27. Wenzel, R.N. Resistance of solid surfaces to wetting by water. Ind. Eng. Chem. 1936, 28, 988-994. [CrossRef]

28. Cassie, A.B.; Baxter, S. Wettability of porous surfaces. Trans. Faraday Soc. 1944, 40, 546-551. [CrossRef]

29. Wang, J.; Chen, S.; Chen, D. Spontaneous transition of a water droplet from the Wenzel state to the Cassie state: A molecular dynamics simulation study. Phys. Chem. Chem. Phys. 2015, 17, 30533-30539. [CrossRef]

30. Li, W.; Ma, X.; Xu, J.; Wang, Y.; Lei, J. Effects of base angle and wettability of nanostructures on droplet wetting behaviors. Acta Phys. Sin. 2021, 1-21. in press. (In Chinese)

31. Saha, J.K.; Matin, M.A.; Jang, J.; Jang, J. Molecular Dynamics Simulation Study on the Wetting Behavior of a Graphite Surface Textured with Nanopillars. Bull. Korean Chem Soc. 2013, 34, 1047-1050. [CrossRef]

32. Bico, J.; Thiele, U.; Quere, D. Wetting of textured surfaces. Colloid Surf. A 2002, 206, 41-46. [CrossRef]

33. Drelich, J.W.; Boinovich, L.; Chibowski, E.; Volpe, C.D.; Hołysz, L.; Marmur, H.A.; Siboni, S. Contact angles: History of over 200 years of open questions. Surf. Innov. 2020, 8, 3-27. [CrossRef]

34. Misawa, H.; Juodkazis, S. 3D Laser Microfabrication: Principles and Applications; Wiley VCH Verl: Weinheim, Germany, 2006; Volume 11, pp. 299-300.

35. Luo, Y.; Jia, W.; Song, Y.; Liu, B. High-repetition-rate femtosecond laser micromachining of poly (methyl methacrylate). Chin. Opt. Lett. 2015, 13, 70003 .

36. Li, J.; Wang, W.; Mei, X.; Sun, X.; Pan, A. The formation of convex microstructures by laser irradiation of dual-layer polymethylmethacrylate (PMMA). Opt. Laser Technol. 2018, 106, 461-468. [CrossRef]

37. Meunier, T.; Villafranca, A.; Bhardwaj, R.; Weck, A. Mechanism for spherical dome and microvoid formation in polycarbonate using nanojoule femtosecond laser pulses. Opt. Lett. 2012, 37, 3168-3170. [CrossRef] [PubMed] 